\title{
The paucity of globular clusters around the field elliptical NGC 7507^
}

\author{
J. P. Caso ${ }^{1,2}$, T. Richtler ${ }^{3}$, L. P. Bassino ${ }^{1,2}$, R. Salinas ${ }^{4}$, R. R. Lane ${ }^{3}$, and A. Romanowsky, \\ ${ }^{1}$ Instituto de Astrofísica de La Plata (CCT La Plata-CONICET-UNLP), Facultad de Ciencias Astronómicas y Geofísicas, \\ Universidad Nacional de La Plata, Argentina \\ e-mail: jpceda@gmail.com \\ 2 Consejo Nacional de Investigaciones Científicas y Técnicas (CONICET), C1033AAJ, Ciudad Autónoma de Buenos Aires, \\ Argentina \\ 3 Departamento de Astronomía, Universidad de Concepción, Casilla 160-C Concepción, Chile \\ ${ }^{4}$ Finnish Centre for Astronomy with ESO, University of Turku, Väisäläntie 20, 21500 Piikkiö, Finland \\ 5 Department of Physics and Astronomy, San José State University, One Washington Square, San Jos, CA 95192, USA \\ ${ }^{6}$ University of California Observatories, 1156 High St., Santa Cruz, CA 95064, USA
}

Received 2 January 2013 / Accepted 3 May 2013

\begin{abstract}
Context. There is strong evidence that globular cluster systems (GCSs) of massive galaxies are largely assembled by infall/accretion processes. Therefore, we expect the GCSs of isolated elliptical galaxies to be poor. Although not completely isolated, NGC 7507 is a massive field elliptical galaxy with an apparently very low dark matter content.

Aims. We determine the richness, the colour distribution, and the structural properties of the GCS of NGC 7507.

Methods. We performed wide-field Washington photometry with data obtained with the MOSAIC II camera at the 4m-Blanco telescope (CTIO, Chile).

Results. The GCS is very poor with $S_{N} \approx 0.6$. We identify three subpopulations with peaks at $(C-T 1)$ colours of $1.21,1.42$, and 1.72. The bluest population may represent the old, metal-poor component. This interpretation is supported by its shallow density profile. The red population is more concentrated, resembling the galaxy light. The intermediate-colour population is strongly peaked in colour, and we interpret this population as the signature of a starburst whose age depends on the metallicity, but should be quite old, since no signatures of a merger are identifiable. In addition, we find a main sequence in the stellar foreground population, which we attribute to the Sagittarius dwarf tidal stream.

Conclusions. The extrordinarily poor GCS of NGC 7507, a massive elliptical galaxy, is an illustration of how important the environmental conditions are for producing rich GCSs.
\end{abstract}

Key words. galaxies: elliptical and lenticular, cD - galaxies: clusters: individual: NGC 7507 - galaxies: star clusters: general

\section{Introduction}

Elliptical galaxies are predominantly found in groups and clusters of galaxies. Isolated elliptical galaxies, on the other hand, are rare and, therefore, mostly distant. The term "isolated ellipticals" dates back to photographic times (Karachentseva 1973), when elliptical galaxies were understood to be featureless, with faint features not easily visible. Recently, Tal et al. (2009) showed that the majority of isolated ellipticals in their sample showed tidal features, which could indicate previous merger activity.

Ellipticals in clusters have accreted a significant part of their material since $z=2$ (van Dokkum et al. 2010), see also Jiménez et al. (2011). The possibility of accreting material is plausibly weaker for isolated galaxies, so this could constitute a major difference between isolated elliptical galaxies and ellipticals in clusters, and could be manifest in their globular cluster systems (see Richtler 2013, for a recent review). Not much is known about globular cluster systems (GCSs) of isolated elliptical galaxies. That the richness of a GCS is related to its

\footnotetext{
* Photometric data are only available at the CDS via anonymous ftp to cdsarc.u-strasbg. fr $(130.79 .128 .5)$ or via http://cdsarc.u-strasbg.fr/viz-bin/qcat?]/A+A/555/A56
}

local galaxy density is not a new insight, however (West 1993). A compilation of GCS properties, including the environmental density of host galaxies, is given in Spitler et al. (2008). The few isolated ellipticals appearing in that study have relatively low specific frequency $\left(S_{N}\right)$ values ${ }^{1}$. Recent work on the isolated ellipticals NGC 5812 and NGC 3585 also revealed very low $S_{N}$ values (Lane et al. 2013). However, this is not always the case, as the sample of galaxies in low-density environments presented in Cho et al. (2012). In this context, NGC 7507 is a particularly interesting object. Its morphology is that of a "real" elliptical galaxy (E0) without clear signs of tidal distortions. Dark matter is not necessarily required to explain its kinematics (Salinas et al. 2012), however, some dark matter may be present, in conjunction with a radial anisotropy of stellar orbits. Even when some dark matter exists, the total dark matter content is much lower than expected for an elliptical galaxy of this mass (Niemi et al. 2010). NGC 7507 has a spiral galaxy companion (NGC 7513) at a projected distance of $18^{\prime}$, and is, therefore, not truly isolated. Here we investigate its globular cluster system using wide-field Washington photometry. This photometric system has proven

1 The specific frequency $S_{N}$ is defined as $S_{N}=N_{\mathrm{GC}} \times 10^{0.4\left(M_{V}+15\right)}$, where $N_{\mathrm{GC}}$ is the total number of globular clusters of a GC system and $M_{V}$ the absolute magnitude of the host galaxy. 


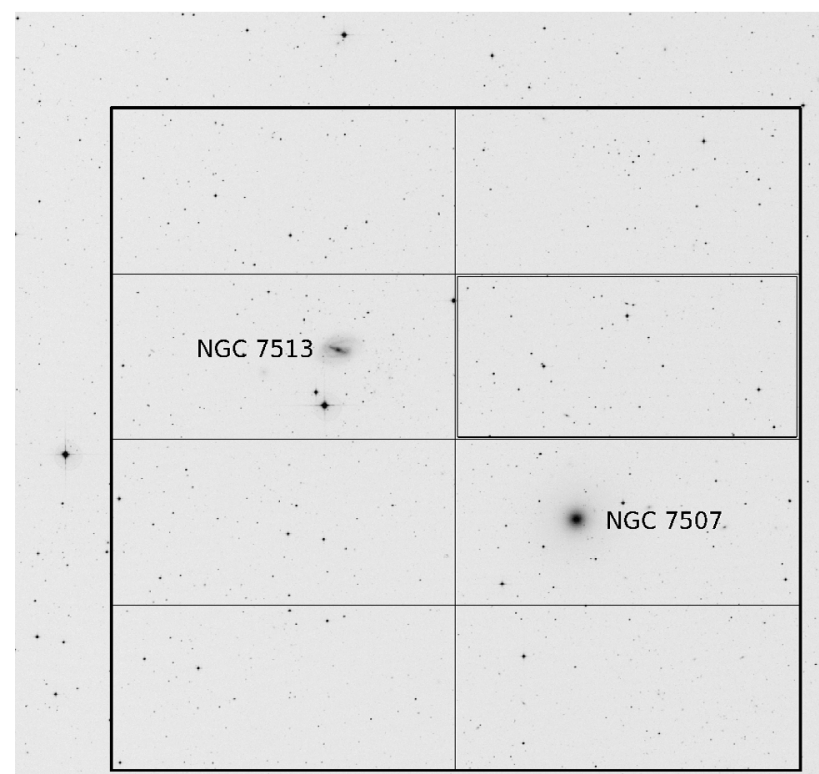

Fig. 1. Our MOSAIC II field around NGC 7507 overlaid onto a DSS image. The field is $36^{\prime} \times 36^{\prime}$. North is up, east to the left. The companion galaxy NGC 7513 is seen toward the north-east. The eight individual CCDs of the MOSAIC II camera are indicated. Chip \#3 is identified by a double line.

particularly useful for investigations of GCS in numerous studies (Dirsch et al. 2003a,b, 2005; Bassino et al. 2006a,b). We adopt a distance of $23.2 \mathrm{Mpc}(m-M=31.83)$. At this distance, $1^{\prime \prime}$ corresponds to $112.5 \mathrm{pc}$, and the absolute magnitude within projected $50 \mathrm{kpc}$ is $M_{R}=-22.64$ according to the photometric model of Salinas et al. (2012), corresponding to a stellar mass of $2 \times 10^{11} M_{\odot}$.

\section{Observations and data reductions}

The observations were performed with the MOSAIC II camera (8 CCDs mosaic imager, 16 amplifiers) mounted at the prime focus of the 4-m Blanco telescope at the Cerro Tololo Inter-American Observatory (CTIO, Chile), during 2005 August 5-6. One pixel of the MOSAIC wide-field camera subtends 0.27 arcsec on the $\mathrm{sky}^{2}$, which corresponds to a field of $36 \times 36 \operatorname{arcmin}^{2}$ or approximately $230 \mathrm{kpc} \times 230 \mathrm{kpc}$ at the adopted distance of NGC 7507. The details of the observations have been given in Salinas et al. (2012), where some of these images were used.

The field is shown in Fig. 1 and was observed with KronCousins $R$ and Washington $C$ filters. We selected the $R$ filter instead of the original Washington $T_{1}$ because Geisler (1996) has shown that the Kron-Cousins $R$ filter is more efficient than $T_{1}$ (see Salinas et al. 2012, for a more thorough explanation). After the calibration, $C$ and $R$ instrumental magnitudes were transformed directly into $C$ and $T_{1}$ standard magnitudes.

The data were dithered to fill in the gaps between the eight individual MOSAIC chips (see Fig. 1 to identify the location of the different chips on the field). Originally, four images in $R$ with exposure times of $720 \mathrm{~s}$ each, and seven images in $C$ with exposures of $1800 \mathrm{~s}$ each were obtained (shorter exposures of $60 \mathrm{~s}$ in $R$ and of $300 \mathrm{~s}$ in $C$ were also obtained to avoid saturation at the galaxy core). However, according to CTIO reports, the first

\footnotetext{
2 http://www.ctio.noao.edu/mosaic
}

night was "useful" and only the second one was "photometric". To improve the photometry, the calibration was revised for the present paper. Moreover, to obtain the final combined images, we used the long-exposure $R$ images, but only five out of the seven $C$, i.e. those observed on the second night with better seeing.

The tasks in the MSCRED package within IRAF were applied to reduce the MOSAIC data, following some of the guides $^{3}$. Due to the large field of the MOSAIC images, the pixel scale across the CCDs is variable. As a consequence, the brightness of point sources may differ by up to $4 \%$ between image centre and corners. The MSCRED package contains tasks to correct for this effect. For instance, the MSCCMATCH task uses a reference celestial catalogue (an astrometric catalogue) to correct for any difference between the reference coordinates and the observed ones, i.e. updates the world coordinate system (WCS) of the image to the WCS of the reference system. In a later stage, the MSCIMATCH task takes into account any possible differences in the intensity scale between the chips, homogenizing them according to the intensities of a reference image. The final combined $R$ image had remaining sensitivity variations of up to $1.4 \%$, the final $C$ image of up to $3.0 \%$, calculated from peakto-peak over the whole images. In these final images, the seeing is $1.1^{\prime \prime}$ and $1.3^{\prime \prime}$ in the $R$ and $C$ frames, respectively.

The chip \#3 (Fig. 1) caused problems during the final stacking of the images, performed with the MSCSTACK task. The regions where chip \#3 overlapped with other CCDs caused multiple images with the dithering procedure. Masking out the respective part of chip \#3 solved the problem, but introduced some inhomogeneity.

\section{Photometry}

To facilitate point source detection, the extended galaxy light was subtracted using a ring median filter (Secker 1995) with an inner radius of $8.0^{\prime \prime}$ and an outer radius of $9.3^{\prime \prime}$. These values were selected according to our previous experience with MOSAIC images (e.g. Dirsch et al. 2003a,b), and it was checked that the median filter had not affected the point source photometry. An initial selection of point sources was obtained with the software SExtractor (Bertin \& Arnouts 1996), using a Gaussian filter on the $C$ image. We considered every group of at least five connected pixels above a threshold of $1.5 \sigma$ as a positive detection (DETECT_MINAREA and DETECT_TRESH parameters, respectively). Effective radii of classical GCs do not usually exceed a few parsecs (e.g. Harris 2009), which implies that at the distance to NGC 7507, GCs are seen as point-sources on MOSAIC II images. To select point sources we used the star/galaxy classifier from SExtractor, through the CLASS_STAR parameter, which takes values close to one for point-sources and close to zero for extended sources. All objects with CLASS_STAR $<0.4$ were rejected and about 14000 pointsources were detected on the whole MOSAIC field.

The photometry was performed using Daophot/IRAF in the usual manner. A second-order variable PSF was built for each final $R$ and $C$ image, employing about one hundred bright stars well distributed over the field. The final point source selection was based on the $\chi$ and sharpness parameters from the ALLSTAR task.

As stated above, the calibration performed by Salinas et al. (2012) was improved for the present paper. Three fields of standard stars selected from the list of Geisler (1996) were used to derive the photometric calibration. The revised set of calibration

\footnotetext{
http://iraf.noao.edu/projects/ccdmosaic/
} 
J. P. Caso et al.: The paucity of globular clusters around the field elliptical NGC 7507

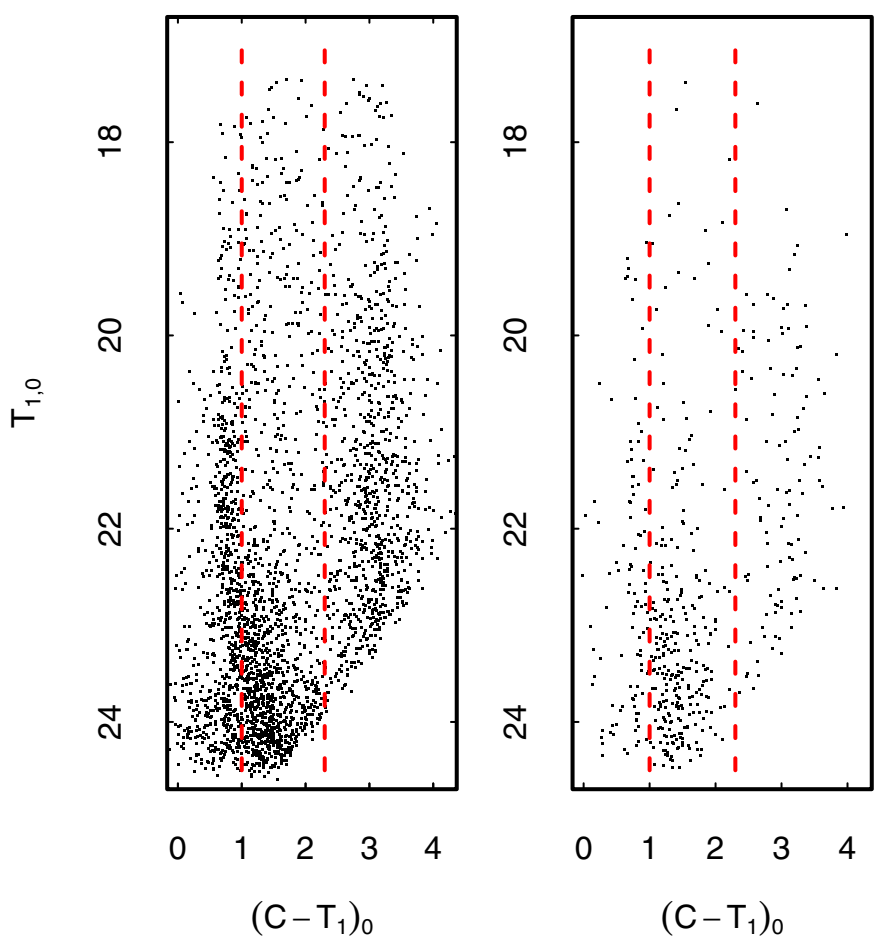

Fig. 2. Colour-magnitude diagram for the point sources in the MOSAIC II field. The left panel shows the entire field, while the right panel shows only objects closer than $7^{\prime}$ to the centre of NGC 7507. The red dashed lines indicate the colour range for old GCs. It is clear that the GCS is quite poor. The main sequence feature is striking, which probably belongs to the Sagittarius stream. See the Appendix for a more detailed discussion.

equations (only $C$ differs from those used by Salinas et al. 2012) is as follows:

$$
\begin{aligned}
T_{1}= & R_{\text {inst }}+(0.89 \pm 0.01)-(0.23 \pm 0.01) X_{\mathrm{R}} \\
& +(0.016 \pm 0.003)\left(C-T_{1}\right) \\
C= & C_{\text {inst }}+(1.54 \pm 0.01)-(0.66 \pm 0.01) X_{\mathrm{C}} \\
& +(0.103 \pm 0.003)\left(C-T_{1}\right)
\end{aligned}
$$

where $R_{\text {inst }}$ and $C_{\text {inst }}$ are the instrumental magnitudes and $X_{\mathrm{R}}$ and $X_{\mathrm{C}}$ are the airmasses, respectively.

The aperture corrections and their corresponding errors, computed from the stars used to build each PSF, are $-0.33 \pm 0.02$ and $-0.32 \pm 0.02$ for the $C$ and $R$ photometry, respectively. The $(B-V)$ reddening was calculated from the Schlegel et al. (1998) maps. The colour excess in the Washington photometric system was adopted as $E_{\left(C-T_{1}\right)}=1.97 \times E_{(B-V)}$ (Harris \& Canterna 1977), and for the $T_{1}$ absorption we used the relation $A_{R} / A_{V}=$ 0.75 (Rieke \& Lebofsky 1985). Thus, the foreground reddening and absorption corrections applied were $E_{\left(C-T_{1}\right)}=0.09$ and $A_{T 1}=0.12$, respectively.

\subsection{Completeness estimation}

To estimate the completeness of our final sample, we added 1000 artificial stars to each final $R$ and $C$ images, equally distributed over the field, covering the magnitude and colour intervals expected for GCs. This process was repeated ten times, reaching a total sample of 10000 artificial stars. The photometry of these new images was performed in the same way as the original.

The completeness function around the galaxy indicates that the $75 \%$ completeness is achieved at $T_{1} \approx 22.3$, and the $40 \%$
Table 1. Completeness up to $7^{\prime}$ from the NGC 7507 centre.

\begin{tabular}{lc}
\hline \hline $\begin{array}{l}T_{1} \\
{[\mathrm{mag}]}\end{array}$ & $\begin{array}{c}\text { Completeness } \\
\%\end{array}$ \\
\hline $19.5-20.0$ & 0.95 \\
$20.0-20.5$ & 0.92 \\
$20.5-21.0$ & 0.92 \\
$21.0-21.5$ & 0.85 \\
$21.5-22.0$ & 0.81 \\
$22.0-22.5$ & 0.76 \\
$22.5-23.0$ & 0.64 \\
$23.0-23.5$ & 0.62 \\
$23.5-24.0$ & 0.58 \\
$24.0-24.5$ & 0.30 \\
\hline
\end{tabular}

at $T_{1} \approx 24$. We adopted $T_{1}=24$ as our magnitude limit. In Table 1 we indicate the completeness for bins of $0.5 \mathrm{mag}$.

\section{Globular cluster system}

\subsection{Selection of globular cluster candidates}

We selected point-sources with $1<\left(C-T_{1}\right)_{0}<2.3$ and $T_{1,0}>20$ as GC candidates. This colour range corresponds to the entire metallicity range for old clusters and is similar to that used in the literature for previous Washington photometric studies of GCs (e.g. Geisler et al. 1996; Dirsch et al. 2003b; Bassino et al. 2006a; Richtler et al. 2012). Figure 2 shows the colour-magnitude diagram (CMD) for all point sources in the entire field (left panel) and for point sources closer than $7^{\prime}$ to the galaxy centre (right panel). We expect the universal turnover-magnitude of old cluster systems at about $m_{V} \approx 24.4$ (see Sect. 4.5). It is clear that this is not a rich GCS. Red dashed lines indicate the $\left(C-T_{1}\right)_{0}$ range for the GC candidates. The left panel shows no sign of a GCS and even for the radius-selected sample, no GCS is apparent.

A striking feature of the CMD is the vertical sequence of objects bluer than $\left(C-T_{1}\right)_{0}=1$. The distribution of these point sources turns out to be uniform over the field.

These sources are accounted for in the general background subtraction. The adopted background region is $\approx 12^{\prime}$ away from NGC 7507, and its area is almost $700 \operatorname{arcmin}^{2}$. That is, the background is far from the projected GCS. In the Appendix, we show that this "strip" is probably a main sequence of a population belonging to the tidal stream of the Sagittarius dwarf galaxy (Ibata et al. 1994).

\subsection{Colour distribution}

Figure 3 shows the background-corrected colour distribution for GC candidates brighter than $T_{1}=24$ in the radial regime $0.5^{\prime}<$ $R<7^{\prime}$ (raw and background counts are listed in Table 2). The outer radial limit is argued for in Sect. 4.3, the inner limit avoids strong incompleteness due to the galaxy brightness. The bin width is 0.05 .

The main observation from Fig. 3 is that the GC colour distribution differs significantly from the "normal" distributions of GCs in giant elliptical galaxies. Most striking is the sharp peak in the colour range $\left(C-T_{1}\right)_{0} \sim 1.40-1.45$.

We fitted the colour distribution by a sum of three Gaussians and obtained $\left(C-T_{1}\right)_{0}=1.21 \pm 0.02,\left(C-T_{1}\right)_{0}=1.42 \pm 0.02$ and $\left(C-T_{1}\right)_{0}=1.72 \pm 0.04$ as mean peak colours. Comparing 


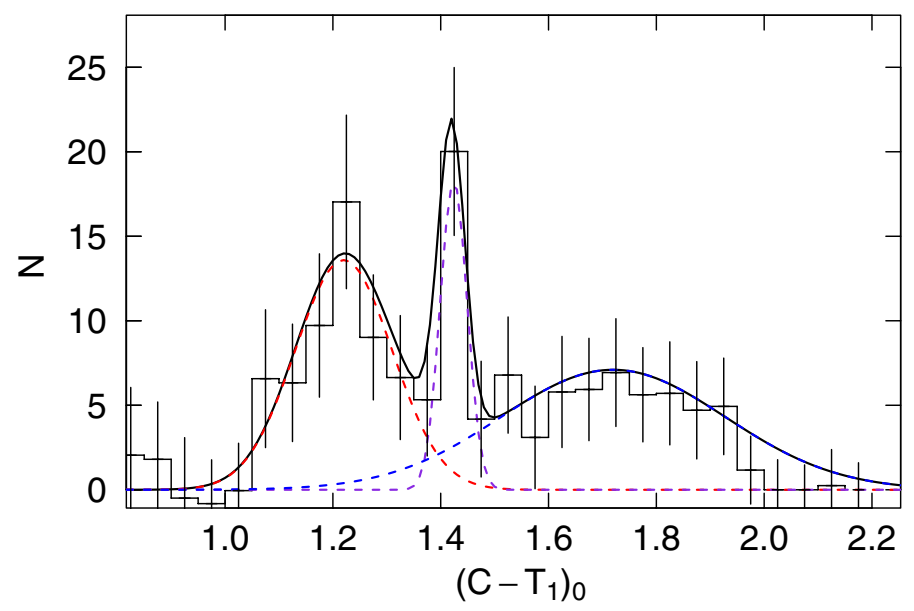

Fig. 3. Background-corrected colour distribution for GC candidates in the radial regime $0.5^{\prime}<R<7^{\prime}$. A sum of three Gaussians has been fitted and is plotted as solid black lines, while each individual Gaussian is plotted as dashed lines. We interpret the sharp peak at $\left(C-T_{1}\right)=1.4$ as the signature of a starburst.

Table 2. Point sources colour distribution.

\begin{tabular}{lcc}
\hline \hline $\begin{array}{l}\left(C-T_{1}\right)_{0} \\
{[\mathrm{mag}]}\end{array}$ & Raw & Background \\
\hline $0.90-0.95$ & 8 & 8 \\
$0.95-1.00$ & 4 & 5 \\
$1.00-1.05$ & 5 & 5 \\
$1.05-1.10$ & 12 & 6 \\
$1.10-1.15$ & 10 & 4 \\
$1.15-1.20$ & 15 & 5 \\
$1.20-1.25$ & 23 & 6 \\
$1.25-1.30$ & 12 & 3 \\
$1.30-1.35$ & 11 & 4 \\
$1.35-1.40$ & 9 & 4 \\
$1.40-1.45$ & 23 & 3 \\
$1.45-1.50$ & 9 & 5 \\
$1.50-1.55$ & 9 & 3 \\
$1.55-1.60$ & 7 & 4 \\
$1.60-1.65$ & 9 & 3 \\
$1.65-1.70$ & 8 & 2 \\
$1.70-1.75$ & 9 & 2 \\
$1.75-1.80$ & 7 & 1 \\
$1.80-1.85$ & 7 & 2 \\
$1.85-1.90$ & 7 & 2 \\
$1.90-1.95$ & 7 & 2 \\
$1.95-2.00$ & 3 & 2 \\
$2.00-2.05$ & 1 & 2 \\
$2.05-2.10$ & 1 & 2 \\
\hline
\end{tabular}

Notes. The first column indicates the bins in which the colour range was divided. The second column shows the raw counts for the colour distribution of GC candidates in the radial regime $0.5^{\prime}<R<7^{\prime}$. The third one shows the background counts, normalized with the ratio between the areas of the science and background regions.

these values with those available in the literature (e.g. Dirsch et al. 2003a; Bassino et al. 2006a,b), the colour of the redder Gaussian is typical of metal-rich GC populations. On the other hand, metal-poor GC populations usually present peak colours in the range $1.25<\left(C-T_{1}\right)_{0}<1.36$, slightly redder than that of the blue population in NGC 7507. However, in Fig. 3 the blue GC candidate distribution seems to be confined to $\left(C-T_{1}\right)_{0}>1$, which is expected for old GCs. The dispersion for the bluest and reddest Gaussians are, $\sigma_{B}=0.08 \pm 0.01$ and $\sigma_{R}=0.20 \pm 0.06$,

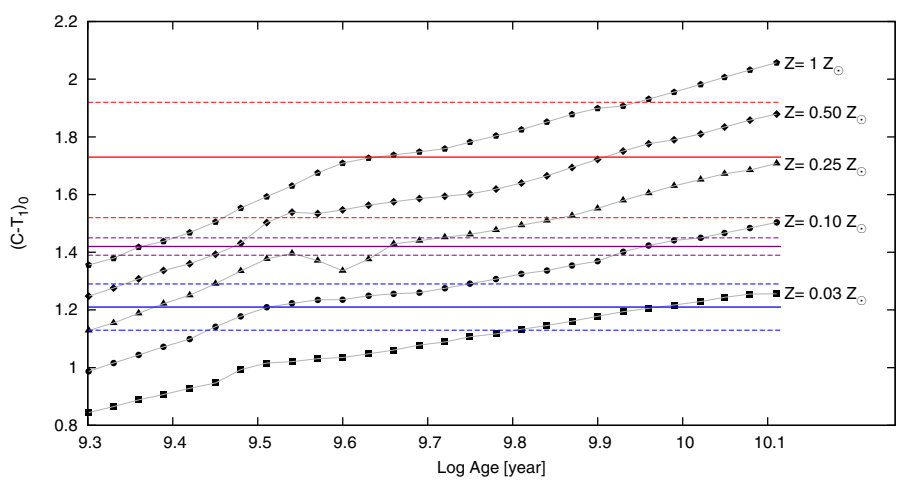

Fig. 4. Theoretical models of single stellar populations by Bressan et al. (2012) for five different metallicities (filled symbols). Blue, violet, and red solid lines indicate the mean colours of the three GC groups, while dashed lines indicate their dispersions.

respectively, which are similar to the values found in other galaxies. The dispersion of the intermediate-colour peak is remarkable. Its dispersion has an upper limit of $0.025 \mathrm{mag}$. This is of the order of the photometric uncertainty and the intrinsic dispersion is therefore most likely even lower.

The authors are aware of only one other example of an intermediate-age starburst being visible in the colour distribution of a GCS, that associated with NGC 1316 (Richtler et al. 2012). Figure 2 in Richtler (2013) shows that spectroscopically confirmed intermediate-age GCs from NGC 1316 are found in a very tight $\left(C-T_{1}\right)_{0}$ range in the colour distribution.

Thus, we have the three GC groups $1<\left(C-T_{1}\right)_{0}<1.37$, $1.37<\left(C-T_{1}\right)_{0}<1.47$ and $1.47<\left(C-T_{1}\right)_{0}<2.2$, which we label blue, intermediate and red samples, respectively. Once the background has been subtracted, each group represents 41, 20 and $39 \%$ of the total number of GC candidates, respectively. The appearance of the colour distribution does not depend strongly on the width of the colour bins. It does depend on the brightness in the sense that the red clusters are mainly faint clusters, which supports the interpretation that the younger and brighter clusters are found among the blue objects.

Figure 4 compares the mean colours of the three groups with theoretical models of single stellar populations by Bressan et al. (2012), using their web-based tool ${ }^{4}$. The models are plotted for five different metallicities (filled symbols), spanning a wide range. Blue, violet, and red solid lines indicate the mean colours of the blue, intermediate and red populations, respectively, while dashed lines indicate their dispersions. If the age estimate for NGC 7507 itself by Salinas et al. (2012) is adopted (i.e. 8-10 Gyr), the metallicities of the blue and red samples are consistent with those of metal-poor and metal-rich old GCs (e.g. Brodie \& Strader 2006).

If the GCs from the intermediate sample have solar metallicity, Fig. 4 indicates an age of about 2-3 Gyr. However, a significant field population of this age does not seem to be present (see the discussion for more details).

\subsection{Projected spatial and radial distributions}

Figure 5 shows the projected spatial distribution of GC candidates in the radial regime $0.5^{\prime}<R<7^{\prime}$, separated by the three different colour intervals defined previously. The circles in each panel represent the limits of the chosen radial regime. Comparing the distribution of the blue (left panel) and

4 http://stev.oapd.inaf.it/cgi-bin/cmd 

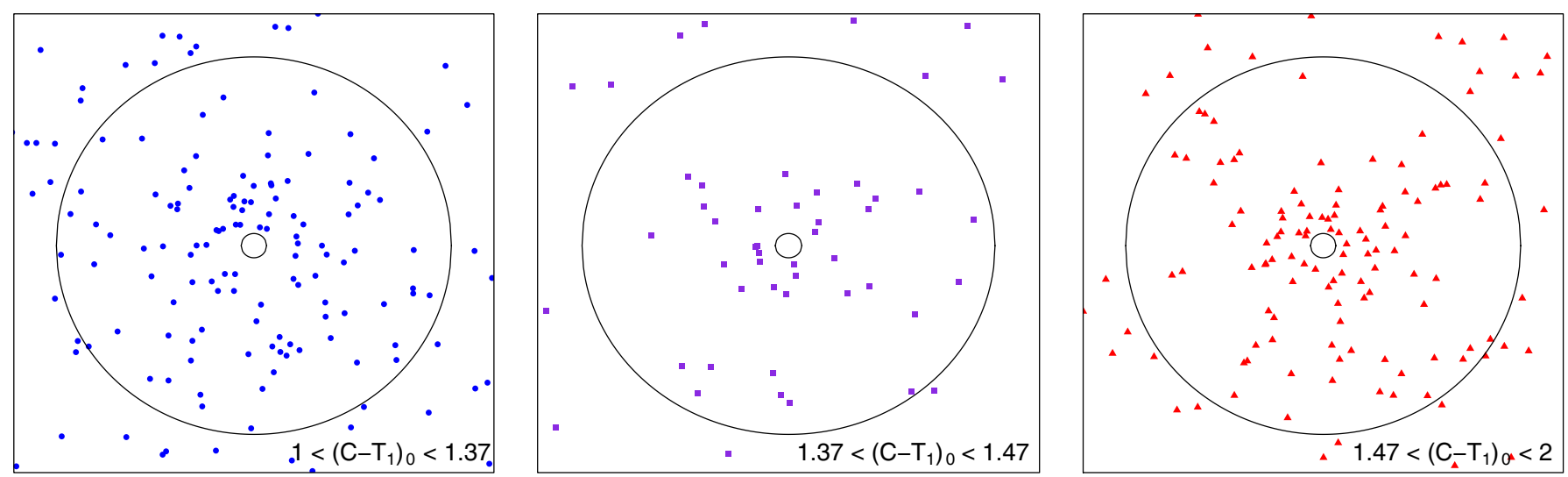

Fig. 5. Projected spatial distribution of GC candidates, separated into three different colour bins. The circles plotted in each panel refer to the selected radial regime for the analysis (i.e. $0.5^{\prime}<R<7^{\prime}$ ). Each figure spans $15.75^{\prime} \times 15.75^{\prime}$. North is up, east to the left.

red sample (right panel), the latter seems to be slightly more concentrated towards the galaxy centre. This is consistent with the usual radial behaviour of metal-poor and metal-rich GCs in elliptical galaxies. The spatial distribution of the intermediate sample (middle panel) seems to prefer smaller radii than the blue sample, and there possibly is a slight preference for the east-west direction, which is lowly defined, however.

The projected radial distribution of the GC candidates are shown in Fig. 6. Panel (a) shows the raw distribution for all GC candidates (open circles) and the background-corrected distributions for the complete colour range (filled black circles) and the blue (blue squares) and red (red triangles) samples. The horizontal dashed line indicates the background level, while the dotted line refers to $30 \%$ of the background level. We propose this limit to define the GCS extension. The dash-dotted line corresponds to $30 \%$ of the background level in the colour ranges of the blue and red samples.

We estimate the extension of the NGC 7507 GCS to approximately $7^{\prime}$ (i.e. $47.25 \mathrm{kpc}$ ). It is not possible to fit a uniform power-law to the distribution of the blue sample because of the two deviating innermost points. These counts cannot be low due to completeness, because the red clusters should have been at least equally affected. In the radial range $1.5^{\prime}-5^{\prime}$ the logarithmic density slopes of the blue and the red sample are indistinguishable. Their values are $-2.4 \pm 0.15$ and $-2.5 \pm 0.2$, respectively.

In the lower panel we compare the radial distributions of the blue and red samples with the light profile of NGC 7507, which is the Hubble-Reynolds profile given by Salinas et al. (2012). An arbitrary scaling was applied for comparison purposes. All slopes are similar, but the deficiency of blue clusters at small radii is striking.

The small size of the intermediate sample prohibits obtaining a radial density profile. Instead, we statistically subtracted the background contribution in the three samples. For this purpose, we introduced seven annuli with a width of $1^{\prime}$ each to cover the full radial range. From each we erased random background objects according to the background density. The mean distance of objects to the galaxy centre was then obtained for each sample. This procedure was repeated one hundred times. Finally, the average of the mean distances and their errors were $3.3 \pm 0.2^{\prime}$, $2.8 \pm 0.3^{\prime}$ and $2.6 \pm 0.2^{\prime}$ for the blue, intermediate and red sample, respectively. A Kolmogorov-Smirnov test (Kolmogorov 1933; Smirnov 1948) was used to compare the radial distribution of the clean intermediate sample with the others for each iteration. In all cases, the probability that blue and intermediate
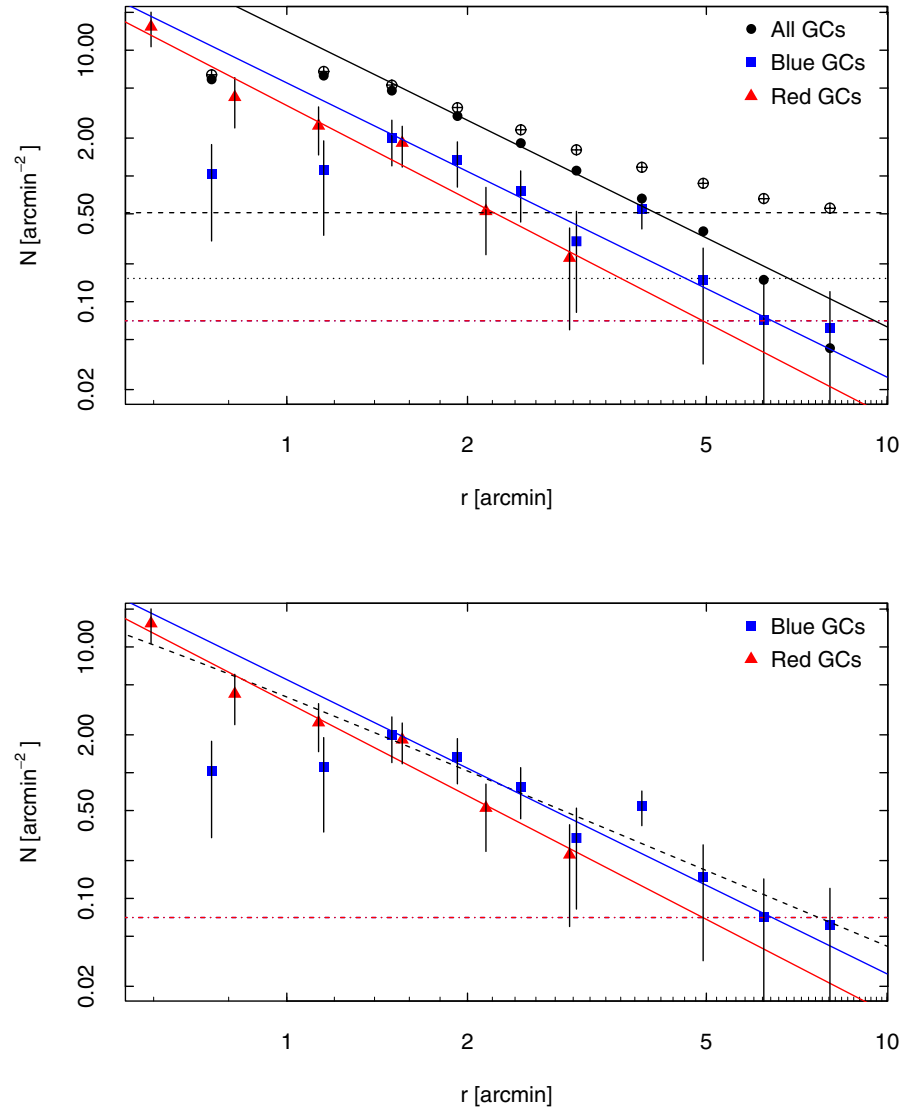

Fig. 6. Upper panel: raw radial distribution for all GC candidates (open circles). Background-corrected distributions for GC candidates with $1<$ $\left(C-T_{1}\right)_{0}<2.3$ (filled black circles) and for the blue (blue squares) and red (red triangles) samples. The solid lines represent power laws (see text) for the respective samples corresponding by colour. The dashed line indicates the background level, while the dotted one refers to the $30 \%$ of the background level. The dash-dotted line is the $30 \%$ of the background level for the blue and red samples, which are indistinguishable. Lower panel: dashed line indicates the Hubble-Reynolds profile of NGC 7507 by Salinas et al. (2012).

samples could be described by the same distribution is almost null. A similar result was obtained when blue and red samples were compared. For the comparison between the intermediate and red samples, a $50 \%$ of probability was obtained. 


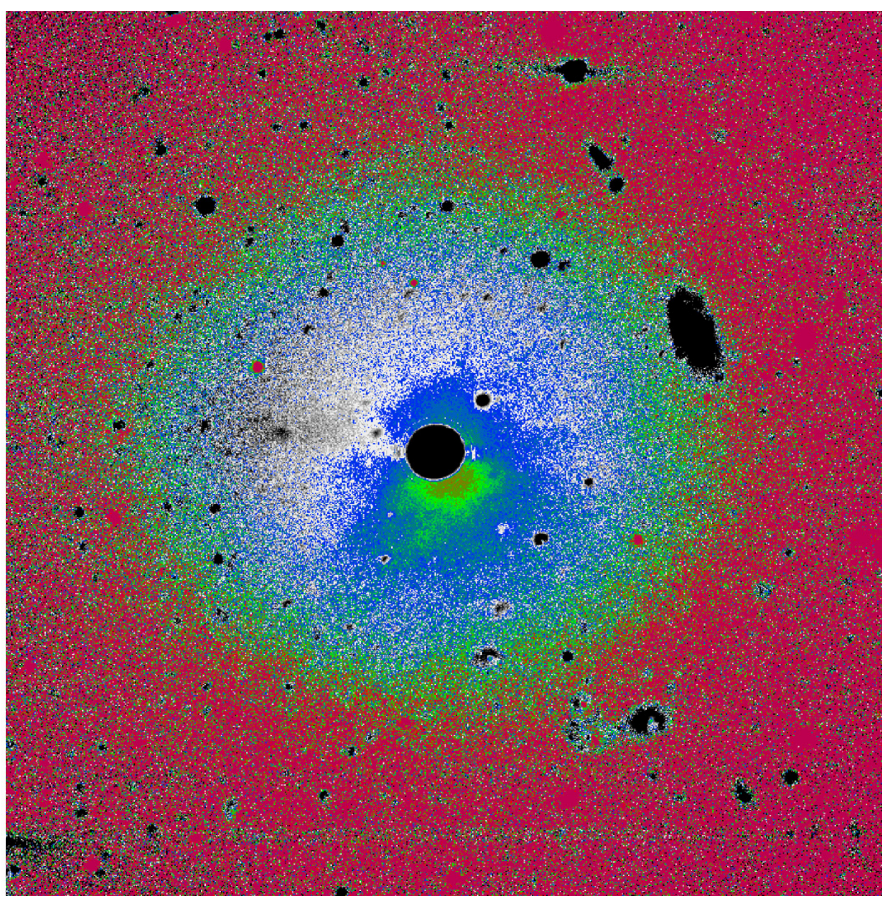

Fig. 7. $4^{\prime} \times 4^{\prime}$ NGC 7507 colour map. North is up, east to the left. The colour palette represents the range $1.85<\left(C-T_{1}\right)<2.2$ from blue to red (beyond a radius of about $1^{\prime}$ the colours are no longer reliable). We interpret the redder parts (indicated with blue and green colours) as being caused by dust, which has been detected before (Temi et al. 2004)

\subsection{Dust in NGC 7507}

Morphologically, NGC 7507 has a smooth appearance without obvious shells or tidal structures (the feature reported by Tal et al. 2009 had not been confirmed by Salinas et al. 2012), except for a central dust lane (Sparks et al. 1985). Salinas et al. (2012) showed the central dust structures in the colour map in short exposure images. The colours are too red to resemble a stellar population and are probably caused by reddening. Here we describe our long-exposure colour map, which shows that there is also dust distributed on a larger scale.

Figure 7 displays a $4^{\prime} \times 4^{\prime}$ NGC 7507 colour map. The colour palette spans the range $1.85<\left(C-T_{1}\right)_{0}<2.2$. It is clear that the colour distribution is not radially symmetric. The redder parts, which are probably caused by reddening due to dust, extend to about $1^{\prime}(6.7 \mathrm{kpc})$ to the south-west. The existence of dust in an otherwise morphologically undisturbed galaxy could be evidence of a past merger and is perhaps related to the existence of the intermediate population of GCs. See the discussion for more remarks.

\subsection{Total numbers and specific frequency}

In this section we estimate the number of members for the NGC 7507 GCS and its specific frequency $S_{N}$. For the radial interval $0.5^{\prime}<R<7^{\prime}$, we counted the background-corrected objects in bins of $0.5 \mathrm{mag}$, down to $T_{1}=24 \mathrm{mag}$, which is our $40 \%$ completeness limit. To correct this for the missing central region, we adopted for the innermost $0.5^{\prime}$ the same surface density as for the first bin, resulting in a correction of five objects (i.e., $\approx 4 \%$ of the $0.5^{\prime}<R<7^{\prime}$ background-corrected sample). This criterion was applied by other authors in GCs studies (e.g., Bassino et al. 2006b), assuming that the radial distribution of GCs might be flatter in the very inner region of a galaxy (e.g., Elson et al. 1998). Finally, we corrected the number of objects in each bin by its corresponding incompleteness, arriving at approximately $210 \mathrm{GC}$ candidates. We still needed to calculate the number of GCs fainter than $T_{1}=24 \mathrm{mag}$. It is expected that an old GC population presents a Gaussian luminosity function with a turn-over magnitude (TOM) in the $V$-band of $M_{\mathrm{TOM}} \approx-7.4$ (e.g. Richtler 2003; Jordán et al. 2007) at our adopted distance at an apparent magnitude of $V \approx 24.4$. The mean $(V-R)$ colour of the GCS is not drastically different from 0.4 , so that our limiting magnitude roughly marks the TOM. This allows us to double the calculated number of GCs brighter than $T_{1}=24$ mag to obtain a total population of approximately $420 \mathrm{GC}$ candidates.

Our photometric model of NGC 7507 (Salinas et al. 2012) gives an absolute brightness of $M_{\mathrm{R}}=-22.64$ (extinction of $0.12 \mathrm{mag}$ included) within $7^{\prime}(47.25 \mathrm{kpc})$ or $M_{\mathrm{V}}=-22.16$ (adopting $(V-R)=0.6$ from Prugniel \& Heraudeau 1998). With this brightness, $S_{N} \approx 0.6$. This value changes with adopted distance, but it will stay far below what is known for elliptical galaxies in cluster environments, even adopting its closest estimate.

\section{Discussion}

\subsection{Total number of GCs and the environment}

The number of GCs around NGC 7507 is only about one tenth of the number of GCs one normally finds around cluster ellipticals of comparable magnitude (e.g. Brodie \& Strader 2006; Richtler 2013).

The comparison with NGC 4636, an elliptical giant galaxy in the outskirts of the Virgo cluster is instructive: while the stellar masses of the galaxies are similar, NGC 4636 has about $6000 \mathrm{GCs}$ (Dirsch et al. 2005). Since the galaxy density around NGC 4636 is not extremely high, this shows that the current environmental density is no very reliable parameter for determining the richness of a GCS.

Still, the literature is not entirely clear in this respect. In Spitler et al. (2008), the few isolated elliptical galaxies indeed have low $S_{N}$-values, but recently Cho et al. (2012) reported on the GCSs of early-type galaxies in low-density environments, some of which appear to have higher $S_{N}$-values, NGC 1172 even with $S_{N}=9.2 \pm 4.4$ (however, we were unable to reproduce this value with the data given in their paper). The conclusion of Cho et al. (2012) that the host galaxy mass is the primary parameter in determining the properties of a GCS, cannot be confirmed with NGC 7507. We moreover remark that our $S_{N}$-value cannot be directly compared with those of Cho et al. (2012), which are systematically too high with respect to our value. The use of the RC3-magnitudes for NGC 7507 for brightness and colour would have meant an absolute brightness of $M_{V}=-21.5$, about 0.7 mag fainter than the brightness of our photometric model.

In addition, Cho et al. (2012) only used the density parameter of Tully (1988) to define a low-density environment. None of the Cho et al. galaxies appear in catalogues of isolated ellipticals (e.g. Reda et al. 2004; Smith et al. 2004; Stocke et al. 2004; Collobert et al. 2006). In particular, NGC 1172 appears as a member of the "NGC 1209 group" according to Sandage (1978).

\subsection{Subpopulations and a possible starburst}

Bimodality is the normal concept for the substructure of the optical colour distribution of giant ellipticals. However, it might 
disappear when using infrared colours (Chies-Santos et al. 2012). Its significance is under discussion (e.g. Richtler 2006; Yoon et al. 2006; Richtler 2013) because the relation between colours and metallicities is unclear. Although we identify three subpopulations, the intermediate population has a special character in that it may have its origin in a starburst younger than the main population. Our blue population does have a bluer peak colour than is known from giant ellipticals (Bassino et al. 2006a).

If we consider the accretion of dwarf galaxies as the dominant process in the assembly of the metal-poor component of GCSs (Cote et al. 1998; Hilker et al. 1999; Peng et al. 2008; Schuberth et al. 2010), there might be a straight forward explanation. In a poor environment with fewer GC donators to accrete, we expect, according to the shape of the galaxy luminosity function, a bias toward faint and, therefore, metal-poor galaxies. As a consequence, the accreted GCs are also more metal-poor and the resulting colour peak may be bluer and the width of the Gaussian more narrow.

For radii larger than $1.5^{\prime}$, no difference of the slopes between red and blue clusters is discernable. However, blue clusters can be followed to larger radii than red clusters. For radii smaller than $1.5^{\prime}$ there is a clear deficiency of blue GCs. This fits to a scenario in which the red (bona fide metal-rich) clusters trace the formation of the bulge population, while the blue (bona fide metal-poor) clusters have mostly been accreted through the accretion of their former host galaxies (see Richtler 2013, for a recent account on the formation of globular cluster systems). This supports the general picture and adds confidence that the blue objects really are the old, metal-poor GC population.

Even if spectroscopic confirmation must have the last word, the peak at $\left(C-T_{1}\right)_{0} \sim 1.4$ seems to indicate a population with a homogeneous age and/or metallicity. The natural origin would be a star burst, triggered by a close encounter, perhaps with NGC 7513, or a merger/infall of a gas-rich galaxy. Another example of a GCS showing peaks in the colour distributions related to starbursts is NGC 1316, where the merger properties are obvious (Richtler et al. 2012). This is not the case for NGC 7507, where shells or tidal tails cannot be identified. To assign an age to the peak colour, one must know the metallicity. At solar metallicity, the age would be about 2-3 Gyr. The existence of a significant field population of this age is not plausible, given the relatively red colour of the galaxy. More probable, therefore, is the hypothesis of a lower metallicity and higher age (see Fig. 4). The infall of a gas-rich dwarf galaxy would be a valid scenario, which could also be responsible for the amount of dust found in NGC 7507. Temi et al. (2004) quotes a mass of $130 \times 10^{4} M_{\odot}$ (their two-component model), which is low compared with starburst galaxies, but definitely shows the existence of dust and the possibility of internal reddening.

\subsection{Dark matter - GC connection}

An interesting suggestion has been made by Spitler et al. (2008). From their compilation of dark halo masses and properties of GCSs, they found that "GCs formed in direct proportion to the halo mass". If we replace "formed" by "appeared", we avoid the underlying suggestion that all GCs form in their respective host galaxies. An important perception of elliptical galaxy evolution is that since $z=2$, the stellar masses of elliptical galaxies in clusters have grown by a factor of about 4 through accretion processes (van Dokkum et al. 2010). Accretion in the $\Lambda \mathrm{CDM}$ context means accretion of galaxies, probably with a wide mass range, including their GCSs and dark matter haloes.
If the rich GCSs of central galaxies grow by accretion, it is plausible that in poor environments with less material to accrete, the GCSs are poor and the dark matter content is low. Salinas et al. (2012) has shown that the kinematics of NGC 7507 can be explained without any requirement for dark matter. However, a small dark-matter halo may be permitted under some radial anisotropy, increasing outwards. The stellar mass of NGC 7507 within $50 \mathrm{kpc}$ is about $2 \times 10^{11} M_{\odot}$, adopting the model of Salinas et al. (2012). Adopting the results of Niemi et al. (2010) as reference for the assignment of dark matter to stellar matter in $\Lambda \mathrm{CDM}$ simulations, one would expect a dark-halo-mass of about $10^{13} M_{\odot}$. This is clearly not supported by the data. A halo mass of about $6 \times 10^{11} M_{\odot}$ could be marginally accomodated.

We can evaluate how NGC 7507 fits in the relation between total mass $M_{180}$ (which is the sum of baryonic and dark mass within a volume with an average density of 180 times the background density) and the mass in GCs, given by Spitler \& Forbes (2009):

$M_{180}=14125 \times M_{\mathrm{GCS}}$

Adopting 420 as the number of clusters and, following Spitler \& Forbes (2009), $4 \times 10^{5} M_{\odot}$ as the average GC mass, we have $1.68 \times 10^{8} M_{\odot}$ as the total mass in GCs. Therefore, one expects $2.4 \times 10^{12} M_{\odot}$ as $M_{180}$. Using $2 \times 10^{11} M_{\odot}$ (1) for the stellar mass, we see that the expected mass is higher by a factor of about 10 than the mass suggested by the observations.

\section{Summary and conclusions}

We investigated the globular cluster system of NGC 7507, a massive elliptical field galaxy.

The globular cluster system of NGC 7507 is very poor, more similar to a spiral galaxy than to a giant elliptical galaxy. For the specific frequency, we derived the extraordinarily low value of $S_{N}=0.6$, which is in line with other galaxies in low-density environments. The colour distribution shows the blue and red peak, commonly described by the term bimodality in the GCSs of elliptical galaxies. The red peak has a similar colour to that found for the GCSs of other host galaxies. However, the blue peak has a colour somewhat bluer than normally found in richer cluster systems, agreeing with other studies of isolated ellipticals (Cho et al. 2012). Another well-known feature is the shallower number density profile of the blue clusters. A peculiarity is a sharp peak at $\left(C-T_{1}\right)=1.4$, making up $20 \%$ of the cluster population.

We suggest the following interpretation:

Owing to its relatively isolated location, only a few accreted dwarf galaxies donated their GCSs to NGC 7507, limiting the metal-poor component. The peak at $(C-T 1)=1.4$ is the signature of a starburst with an a priori unknown age. The most probable hypothesis is the infall/merger of a gas-rich dwarf galaxy. This could have happened 7-8 Gyr ago and would be consistent with a younger age, independently indicated by the low $M / L_{R}$-ratio of 3 (Salinas et al. 2012).

Furthermore, because the dark matter content of NGC 7507 is low, one may see it as an example supporting the relation reported in Spitler et al. (2008), where GCs appear to be roughly in proportion to the dark halo mass.

Acknowledgements. J.P.C. and L.P.B. acknowledge financial support by grants from Universidad Nacional de La Plata and Consejo Nacional de Investigaciones Científicas y Técnicas CONICET (Argentina). T.R. acknowledges financial support from the Chilean Center for Astrophysics, FONDAP Nr. 15010003, from FONDECYT project Nr. 1100620, and from the BASAL Centro de Astrofisica y Tecnologias Afines (CATA) PFB-06/2007. R.R.L. gratefully acknowledges financial support from FONDECYT, project No. 3130403. 


\section{Appendix A: Sagittarius dwarf galaxy tidal tail in the foreground of NGC 7507}

It is apparent that there is an unusual sequence in the CMD of point sources from the NGC 7507 field (Fig. 2), with $T_{1,0} \gtrsim 20.0$ and $0.7 \lesssim\left(C-T_{1}\right)_{0} \lesssim 1.8$. This feature is reminiscent of CMD sequences ascribed to both the Monoceros Ring, an apparently extra-Galactic ring of tidal debris encircling the disc (e.g. Conn et al. 2007, 2008, 2012), and to detections of the Sagittarius (Sgr) dwarf (Ibata et al. 1994) tidal stream (e.g. Mateo et al. 1996; Majewski et al. 1999). Furthermore, the latitude of this field is too high $\left(b \sim-68^{\circ}\right)$, and has a magnitude range that is much too narrow to be attributed to the Galactic disc or any other known Galactic substructure.

Interestingly, this field is located at (RA, Dec) $\left(23^{\mathrm{h}} 12^{\mathrm{m}},-28^{\circ} 32^{\prime \prime}\right)$, a location well-placed to include stellar members of the tidal stream from the Sgr dwarf. The colour of the vertical component of the CMD sequence $\left(\left[C-T_{1}\right] \sim 0.7\right.$, Fig. 2) correlates well with the $(V-I)$ colour of the same part of the sequence $([V-I] \sim 0.8$; Mateo et al. 1996; Majewski et al. 1999), assuming a metalliticy of $Z=0.08$ (colour transformations derived following Bressan et al. 2012 with corrections by Girardi et al. 2010). Because of the obvious similarities between the CMD sequence shown in Fig. 2, the location of the field and the colour of the sequence, we attribute this feature to a detection of the Sgr tidal stream in the foreground of NGC 7507. Figure A.1 shows the colour distribution of the point sources from the background region, scaled down to the size of the galaxy field. According to the CMD shown in Fig. 2, the bins with the highest counts are located in the colour range $0.7 \lesssim\left(C-T_{1}\right)_{0} \lesssim 1$. For higher colour values, redder bins present lower count values. The dashed lines indicate the mean colours of the three Gaussians fitted to the GC colour distribution in Sect. 4.2. The counts in the range $1 \lesssim\left(C-T_{1}\right)_{0} \lesssim 2.2$ imply that the three peaks in the GC colour distribution would persist even if the contamination were increased by $50 \%$.

A comparison with recent results from Pan-STARRS1 (Slater et al. 2013) shows that the line-of-sight toward NGC 7507 crosses the bright arm in the southern part of the Sgr stream.

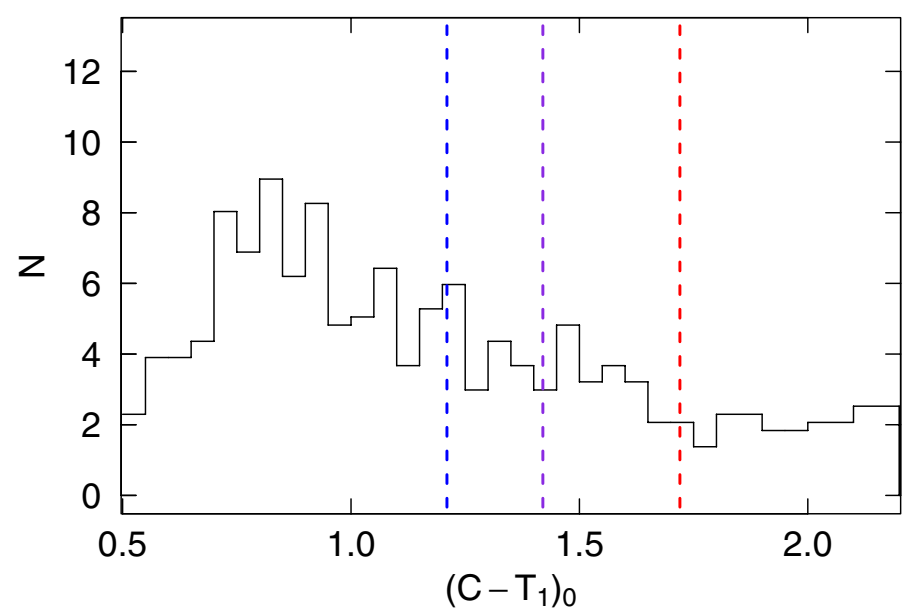

Fig. A.1. Colour distribution of the point sources in the background region, scaled down to the size of the galaxy field. The dashed lines indicate the mean colours of the three gaussians fitted to the GC colour distribution in Sect. 4.2, $\left(C-T_{1}\right)_{0}=1.21,\left(C-T_{1}\right)_{0}=1.42$ and $\left(C-T_{1}\right)_{0}=1.72$, respectively.
The CMD sequence broadens toward dimmer magnitudes. Because photometry is intrinsically more uncertain at fainter magnitudes, this broadening is most likely due to uncertainties in the photometry, and therefore does not reflect a stream with a large systemic distance spread between stellar stream members.

\section{References}

Bassino, L. P., Faifer, F. R., Forte, J. C., et al. 2006a, A\&A, 451, 789 Bassino, L. P., Richtler, T., \& Dirsch, B. 2006b, MNRAS, 367, 156 Bertin, E., \& Arnouts, S. 1996, A\&AS, 117, 393

Bressan, A., Marigo, P., Girardi, L., et al. 2012, MNRAS, 427, 127

Brodie, J. P., \& Strader, J. 2006, ARA\&A, 44, 193

Chies-Santos, A. L., Larsen, S. S., Cantiello, M., et al. 2012, A\&A, 539, A54

Cho, J., Sharples, R. M., Blakeslee, J. P., et al. 2012, MNRAS, 422, 3591

Collobert, M., Sarzi, M., Davies, R. L., Kuntschner, H., \& Colless, M. 2006, MNRAS, 370, 1213

Conn, B. C., Lane, R. R., Lewis, G. F., et al. 2007, MNRAS, 376, 939

Conn, B. C., Lane, R. R., Lewis, G. F., et al. 2008, MNRAS, 390, 1388

Conn, B. C., Noël, N. E. D., Rix, H.-W., et al. 2012, ApJ, 754, 101

Cote, P., Marzke, R. O., \& West, M. J. 1998, ApJ, 501, 554

Dirsch, B., Richtler, T., \& Bassino, L. P. 2003a, A\&A, 408, 929

Dirsch, B., Richtler, T., Geisler, D., et al. 2003b, AJ, 125, 1908

Dirsch, B., Schuberth, Y., \& Richtler, T. 2005, A\&A, 433, 43

Elson, R. A. W., Grillmair, C. J., Forbes, D. A., et al. 1998, MNRAS, 295, 240

Geisler, D. 1996, AJ, 111, 480

Geisler, D., Lee, M. G., \& Kim, E. 1996, AJ, 111, 1529

Girardi, L., Williams, B. F., Gilbert, K. M., et al. 2010, ApJ, 724, 1030

Harris, H. C., \& Canterna, R. 1977, AJ, 82, 798

Harris, W. E. 2009, ApJ, 699, 254

Hilker, M., Infante, L., \& Richtler, T. 1999, A\&AS, 138, 55

Ibata, R. A., Gilmore, G., \& Irwin, M. J. 1994, Nature, 370, 194

Jiménez, N., Cora, S. A., Bassino, L. P., Tecce, T. E., \& Smith Castelli, A. V. 2011, MNRAS, 417, 785

Jordán, A., McLaughlin, D. E., Côté, P., et al. 2007, ApJS, 171, 101

Karachentseva, V. E. 1973, Astrofizicheskie Issledovaniia Izvestiya Spetsial'noj Astrofizicheskoj Observatorii, 8, 3

Kolmogorov, A. 1933, Giornale dell' Istituto Italiano degli Attuari, 4, 83

Lane, R. R., Salinas, R., \& Richtler, T. 2013, A\&A, 549, A148

Majewski, S. R., Siegel, M. H., Kunkel, W. E., et al. 1999, AJ, 118, 1709

Mateo, M., Mirabal, N., Udalski, A., et al. 1996, ApJ, 458, L13

Niemi, S.-M., Heinämäki, P., Nurmi, P., \& Saar, E. 2010, MNRAS, 405, 477

Peng, E. W., Jordán, A., Côté, P., et al. 2008, ApJ, 681, 197

Prugniel, P., \& Heraudeau, P. 1998, A\&AS, 128, 299

Reda, F. M., Forbes, D. A., Beasley, M. A., O’Sullivan, E. J., \& Goudfrooij, P. 2004, MNRAS, 354, 851

Richtler, T. 2003, Lect. Notes phys., 635, 281

Richtler, T. 2006, Bull. Astron. Soc. India, 34, 83

Richtler, T. 2013, ASP Conf. Ser., 470, 327

Richtler, T., Bassino, L. P., Dirsch, B., \& Kumar, B. 2012, A\&A, 543, A131

Rieke, G. H., \& Lebofsky, M. J. 1985, ApJ, 288, 618

Salinas, R., Richtler, T., Bassino, L. P., Romanowsky, A. J., \& Schuberth, Y. 2012, A\&A, 538, A87

Sandage, A. 1978, AJ, 83, 904

Schlegel, D. J., Finkbeiner, D. P., \& Davis, M. 1998, ApJ, 500, 525

Schuberth, Y., Richtler, T., Hilker, M., et al. 2010, A\&A, 513, A52

Secker, J. 1995, PASP, 107, 496

Slater, C. T., Bell, E. F., Schlafly, E. F., et al. 2013, ApJ, 762, 6

Smirnov, N. 1948, Ann. Math. Stat., 19, 279

Smith, R. M., Martínez, V. J., \& Graham, M. J. 2004, ApJ, 617, 1017

Sparks, W. B., Wall, J. V., Thorne, D. J., et al. 1985, MNRAS, 217, 87

Spitler, L. R., \& Forbes, D. A. 2009, MNRAS, 392, L1

Spitler, L. R., Forbes, D. A., Strader, J., Brodie, J. P., \& Gallagher, J. S. 2008, MNRAS, 385, 361

Stocke, J. T., Keeney, B. A., Lewis, A. D., Epps, H. W., \& Schild, R. E. 2004, AJ, 127, 1336

Tal, T., van Dokkum, P. G., Nelan, J., \& Bezanson, R. 2009, AJ, 138, 1417

Temi, P., Brighenti, F., Mathews, W. G., \& Bregman, J. D. 2004, ApJS, 151, 237

Tully, R. B. 1988, Nearby galaxies catalog (Cambridge University Press)

van Dokkum, P. G., Whitaker, K. E., Brammer, G., et al. 2010, ApJ, 709, 1018

West, M. J. 1993, MNRAS, 265, 755

Yoon, S.-J., Yi, S. K., \& Lee, Y.-W. 2006, Science, 311, 1129 